\title{
Análisis de factores moduladores de la intervención psicosocial en preescolares con trastorno por déficit de atención/hiperactividad
}

\author{
Rebeca Siegenthaler-Hierro, María Jesús Presentación-Herrero, Carla Colomer-Diago, Ana Miranda-Casas
}

Introducción. Aunque el diagnóstico del trastorno por déficit de atención/hiperactividad (TDAH) en preescolares es cada vez más frecuente, se sabe relativamente poco acerca de su tratamiento en este período evolutivo. Algunos efectos adversos de la intervención farmacológica no aconsejan su uso como primera opción de intervención en estas edades.

Objetivo. Analizar la eficacia de las intervenciones psicosociales para responder tempranamente a las necesidades de niños con TDAH, especialmente las enmarcadas en los modelos cognitivoconductuales y socioconstructivistas.

Desarrollo. La revisión de las investigaciones realizadas en los últimos años en relación con este tema destaca la eficacia de los programas de entrenamiento a padres, solos o combinados con intervenciones en la escuela y con los propios niños. La comunicación entre padres y profesores se ha mostrado como factor determinante del éxito de dichos programas. Otro aspecto que parece propiciar la eficacia del tratamiento en esta etapa educativa es la inclusión dentro del currículo escolar de actividades mediadas encaminadas a desarrollar la autorregulación.

Conclusiones. En preescolares con TDAH, una actuación preventiva basada en estos modelos, que incremente la intensidad de la intervención en función de la respuesta del alumno, podrá evitar problemas futuros.

Palabras clave. Cognitivoconductual. Factores moduladores. Intervención psicosocial. Preescolares. Socioconstructivismo. TDAH.

\section{Introducción y objetivo}

Pese a la falta de criterios claros y de instrumentos de evaluación apropiados, el diagnóstico del trastorno por déficit de atención/hiperactividad (TDAH) en niños en edad preescolar es cada vez más frecuente, con prevalencias del 0,3-6,5\% [1]. Conocemos también que el TDAH se caracteriza ya en fases tempranas por altas tasas de comorbilidad, especialmente con el trastorno negativista desafiante (52,2\%) [2], aunque se sabe relativamente poco acerca de su tratamiento en este período evolutivo.

Actualmente, coexisten dos modalidades de intervención. La primera, médica, consiste en la administración de fármacos que han mostrado su utilidad, especialmente en niños mayores con TDAH. Con niños de educación infantil, las revisiones de estudios metodológicamente rigurosos concluyen que el metilfenidato, especialmente, es efectivo para tratar los síntomas del TDAH, así como para mejorar la conducta y la realización de los trabajos de clase $[3,4]$. No obstante, los datos indican también que los niños pequeños son más sensibles a los efectos adversos, con un incremento de la irritabilidad, dolores de cabeza, cambios de humor, inhibición, letargo, problemas de sueño, reducción del apetito y disminución de la tendencia esperada en el aumento de peso y de altura [5,6]. Otro problema añadido, no menor, es la falta de información de los efectos de los psicoestimulantes sobre el desarrollo cerebral de niños de educación infantil [4]. Esto hace que la administración de fármacos no acostumbre a ser la primera opción de intervención recomendada en preescolares con TDAH. La segunda aproximación, de corte psicosocial, pretende responder tempranamente de forma eficaz a las necesidades específicas de los preescolares con TDAH, fundamentalmente desde modelos cognitivoconductuales y socioconstructivistas.

Este trabajo pretende analizar la eficacia de las intervenciones psicosociales para responder tempranamente a las necesidades de niños con TDAH, especialmente aquéllas enmarcadas en los modelos cognitivoconductuales y socioconstructivistas.

\section{Intervenciones con un enfoque cognitivoconductual}

Su objetivo es proporcionar a las personas que interactúan con los niños pautas de actuación que potencien conductas adaptativas, y a los niños estrate-
Universitat Jaume I; Castellón (R. Siegenthaler-Hierro, M.J. Presentación-Herrero). Universitat de València; Valencia, España (C. Colomer-Diago,

A. Miranda-Casas).

Correspondencia: Dra. Ana Miranda Casas. Departamento de Psicología Evolutiva y de la Educación. Universitat de València. Avda. Blasco Ibáñez, 21. E-46010 Valencia.

Fax: $+34963983880$

E-mail:

ana.miranda@uv.es

Financiación: Financiado por el MICINN (ref.: BES-2010-033019)

Declaración de intereses: Los autores declaran que no hay conflicto de intereses para la publicación de este trabajo.

Aceptado tras revisión externa: 07.01.13.

Cómo citar este artículo: Siegenthaler-Hierro R, PresentaciónHerrero MJ, Colomer-Diago C, Miranda-Casas A. Análisis de factores moduladores de la intervención psicosocial en preescolares con trastorno por déficit de atención/hiperactividad. Rev Neurol 2013; 56 (Supl 1): S85-92.

(c) 2013 Revista de Neurología 
gias y habilidades que les ayuden a regular su propia conducta. Sobresalen, por su probada utilidad, los programas de entrenamiento a padres, los programas para profesores con acomodaciones en el contexto del aula, y las intervenciones con los niños para mejorar su autorregulación cognitiva y socioemocional [3,4,7-11].

\section{Intervención con los padres}

Pretende fomentar el desarrollo de habilidades parentales que promuevan interacciones positivas padre-hijo, mediante la enseñanza de estrategias para el establecimiento de normas y hábitos, y el manejo de comportamientos negativos [3,11]. Entre los programas destacan el Triple P Positive Parenting Programme [12] y el Incredible Years Parent Training Programme [13].

El primero [12,14] comprende 10 sesiones de una hora con un experto. La sesión 1 versa sobre las causas de los problemas de conducta en la infancia. Las sesiones 2 y 3 se centran en 17 estrategias básicas de manejo del niño: 10 para promover el desarrollo, como la alabanza o la atención, y siete para promover el establecimiento de límites efectivos, como introducción de normas, instrucciones claras o tiempo fuera. Las sesiones 4, 5 y 6 son sesiones en casa, en las que se observa a los padres interactuando con sus hijos e implementando las habilidades aprendidas. En las sesiones 7, 8, 9 y 10 se desarrollan estrategias para promover la generalización y el mantenimiento mediante la programación de actividades fuera del hogar. La metodología del programa incluye modelado, desempeño de papeles, retroalimentación y deberes.

Existe una versión mejorada que incrementa el programa estándar con dos sesiones adicionales sobre comunicación de pareja (habilidades para mejorar su relación, el trabajo en equipo y la coherencia en el uso de estrategias) y habilidades de afrontamiento (para ayudar a los padres con dificultades personales que interfieren con sus habilidades parentales, como depresión o estrés). Los estudios realizados con este programa con preescolares con TDAH o problemas de conducta (Tabla I) ponen de manifiesto que ambas versiones producen similares reducciones en problemas de conducta y mejoras en competencia parental, que se mantienen un año después de concluidos los tratamientos [15-17].

El programa de entrenamiento a padres Incredible Years $[18,19]$ se implementa con grupos de 12 16 padres, en sesiones de dos horas semanales, durante 20 semanas. Incluye la enseñanza de las siguientes habilidades: establecimiento de una rela- ción positiva con los hijos a través del juego y de actividades centradas en el niño; uso de la alabanza, el refuerzo y los incentivos; uso del establecimiento de límites, instrucciones claras y rutinas; y uso de estrategias de disciplina (time-out). Los padres aprenden estas habilidades a través de la discusión en grupo, tormenta de ideas, desempeño de papeles, solución de problemas y deberes. También se utiliza el modelado por vídeo mediante la visualización de situaciones comunes a las que se enfrentan los padres. Tras la presentación de los vídeos, se debate y se buscan las soluciones más adecuadas a los problemas planteados. El programa reduce los problemas de conducta y mejora las habilidades parentales en el manejo de los hijos preescolares con problemas de conducta $[20,21]$. Además, disminuye los niveles de inatención e hiperactividad/impulsividad valoradas por los padres, con un 52\% de cambio clínicamente significativo (Tabla I). Las mejoras en sintomatología básica de TDAH son igualmente apreciables incluso tras controlar las mejoras en problemas de conducta [13].

\section{Intervención en el contexto escolar}

Los escasos programas desarrollados con preescolares se han mostrado eficaces para mejorar los problemas de conducta en el aula y reducir la sintomatología de TDAH [22]. Sin embargo, su utilidad para potenciar las habilidades curriculares no es tan clara.

Esta cuestión se contempla en el trabajo de Kern et al [22], en el que se aplicó un paquete de intervención temprana durante seis meses a niños de 3 a 5 años con riesgo de TDAH y problemas de conducta (Tabla I). El programa incluía entrenamiento en técnicas de modificación de conducta, solución de problemas y habilidades preacadémicas de lengua y matemáticas. Como esperaban, los investigadores encontraron reducciones en problemas de conducta y mejoras en el funcionamiento social, y también en las habilidades preacadémicas. Y lo que es más interesante, estudios de seguimiento realizados 12 y 24 meses después mostraron diferencias en el rendimiento preacadémico en función de las respuestas al tratamiento [23,24]. Así, los niños que mejoraron en conducta oposicionista se diferenciaban de los que no lo hicieron en distintas variables de prelectura y matemáticas 12 meses después, aunque pocas fueron las diferencias que persistieron a los 24 meses. Por otro lado, los niños que respondieron positivamente al tratamiento según índices de TDAH mantuvieron las diferencias con los que no lo hicieron sólo en una medida (numerosidad). 
Tabla I. Características y resultados de las diferentes intervenciones con un enfoque cognitivoconductual para preescolares con TDAH.

\begin{tabular}{|c|c|c|c|c|c|c|}
\hline & Programa & Ámbito/edad & Agentes/duración & Contenidos & Metodología & Resultados \\
\hline $\begin{array}{l}\text { Barkley } \\
\text { et al [25] }\end{array}$ & $E P A, I A$ & $\begin{array}{l}\text { Riesgo de } \\
\text { TDAH, PC } \\
4-6 \text { años }\end{array}$ & $\begin{array}{l}\text { Padres, maestros, } \\
\text { padres/maestros } \\
10 \text { semanas }\end{array}$ & $\begin{array}{l}\text { EPA: MC } \\
\text { IA: MC, HS, autocontrol, } \\
\text { control de la ira, } \\
\text { modificaciones } \\
\text { instruccionales }\end{array}$ & $\begin{array}{l}\text { EPA: discusión, desempeño } \\
\text { de papeles, tormenta de ideas, } \\
\text { modelado, deberes } \\
\text { IA: tarjeta casa-escuela, discusión, } \\
\text { modelado, desempeño de } \\
\text { papeles, retroalimentación }\end{array}$ & $\begin{array}{l}\text { EPA: sin mejoras. Baja asistencia de los padres } \\
\text { EPA + IA: } \uparrow \text { conducta adaptativa, } \\
\text { habilidades sociales, atención, autocontrol } \\
\downarrow \text { agresión, conducta externalizante en el aula } \\
\text { Las mejoras desaparecen [26] }\end{array}$ \\
\hline $\begin{array}{l}\text { Kern } \\
\text { et al [22] }\end{array}$ & $\mathrm{EPA}, \mathrm{MCl}$ & $\begin{array}{l}\text { Riesgo de } \\
\text { TDAH, PC } \\
\text { 3-5 años }\end{array}$ & $\begin{array}{l}\text { Padres, } \\
\text { padres/maestros } \\
20 \text { sesiones }\end{array}$ & $\begin{array}{l}\text { EPA: MC, desarrollo } \\
\text { socioemocional y autoestima } \\
\text { MCI: MC, HS, modificaciones } \\
\text { instruccionales, solución } \\
\text { de problemas, habilidades } \\
\text { preacadémicas en lengua } \\
\text { y matemáticas, economía } \\
\text { de fichas }\end{array}$ & $\begin{array}{l}\text { EPA: modelado, vídeos, } \\
\text { discusión, desempeño de papeles, } \\
\text { presentaciones didácticas y } \\
\text { observación } \\
\text { MCI: EPA (padres) + instrucción } \\
\text { directa, modelado, práctica y } \\
\text { retroalimentación, observación } \\
\text { (maestros) }\end{array}$ & $\begin{array}{l}\downarrow \text { problemas de conducta (ambos grupos) } \\
\uparrow \text { funcionamiento social y habilidades } \\
\text { preacadémicas (ambos grupos) } \\
\text { No hay diferencias entre las dos intervenciones } \\
\text { Las mejoras se mantienen tras un año [23] }\end{array}$ \\
\hline $\begin{array}{l}\text { Hoath y } \\
\text { Sanders [16] }\end{array}$ & $\begin{array}{l}\text { Triple P } \\
\text { (EPA) }\end{array}$ & $\begin{array}{l}\text { TDAH } \\
\text { 5-9 años }\end{array}$ & $\begin{array}{l}\text { Padres } \\
5 \text { sesiones }\end{array}$ & $\begin{array}{l}\text { Mejorado: MC, autoevaluación } \\
\text { reforzada, comunicación } \\
\text { con la pareja, habilidades } \\
\text { de afrontamiento }\end{array}$ & $\begin{array}{l}\text { Modelado, desempeño de } \\
\text { papeles, retroalimentación, } \\
\text { deberes }\end{array}$ & $\begin{array}{l}\downarrow \text { problemas de conducta } \\
\uparrow \text { prácticas disciplinarias } \\
\text { y autoeficacia de los padres } \\
\text { Las mejoras se mantienen tras tres meses }\end{array}$ \\
\hline $\begin{array}{l}\text { Jones } \\
\text { et al [13] }\end{array}$ & IY (EPA) & $\begin{array}{l}\text { TDAH o PC } \\
\text { 3-4 años }\end{array}$ & $\begin{array}{l}\text { Padres } \\
12 \text { semanas }\end{array}$ & $\begin{array}{l}\text { MC, resolución de problemas, } \\
\text { relaciones positivas }\end{array}$ & $\begin{array}{l}\text { Discusión, tormenta de ideas, } \\
\text { desempeño de papeles, deberes, } \\
\text { modelado a través de vídeos }\end{array}$ & $\begin{array}{l}\downarrow \text { sintomatología de TDAH } \\
\text { y problemática del niño }\end{array}$ \\
\hline
\end{tabular}

ED: Escuela dinosaurio; EPA: entrenamiento a padres; HS: habilidades sociales; IA: intervención en el aula; IY: Incredible Years; MC: modificación de la conducta. MCI: Multicomponent Intervention; Triple P: Positive Parenting Program; PC: problemas de conducta; TDAH: trastorno por déficit de atención/hiperactividad.

El estudio de Kern et al [22,23] comparó también la eficacia de dos modalidades de tratamiento, intervención con padres únicamente e intervención multicontextual, obteniendo mejoras similares en ambos casos. Estos resultados, que cuestionan la eficacia de la intervención en la escuela y en otros contextos diferentes al familiar, contrastan con los encontrados por Barkley et al [25] al comparar tres condiciones de intervención distintas: en el colegio, con los padres y combinada, y una cuarta condición de control sin ningún tipo de intervención. Participaron en el estudio 158 niños de educación infantil con altos niveles de agresividad, hiperactividad, impulsividad y conducta inatenta. 
Los resultados mostraron que las dos condiciones de intervención escolar, con y sin intervención con padres, produjeron mejoras significativas en múltiples dominios. En concreto, mejoraron las evaluaciones de los padres y los profesores sobre la conducta adaptativa, la atención, el autocontrol y las habilidades sociales de los niños. También se observó la misma tendencia en las observaciones directas de la conducta externalizante en clase. Sin embargo, el tratamiento a padres no produjo mejoras significativas, probablemente debido a su baja participación e implicación en la intervención [25]. En un seguimiento realizado dos años después, se comprobó que todas las mejoras habían desaparecido [26].

\section{Entrenamiento a los niños}

Entre las intervenciones cognitivoconductuales que se han centrado en enseñar a preescolares con TDAH habilidades que les ayuden a autorregularse destaca el programa 'Piensa en voz alta' [27]. El entrenamiento se realiza mediante el empleo de cuatro autoinstrucciones: ¿cuál es mi trabajo?, ¿cómo puedo hacerlo?, ¿estoy utilizando mi plan? y ¿cómo lo hice? Estas autoinstrucciones potencian el pensamiento secuencial, ayudando al niño a comprender las situaciones, a generar las estrategias y mediadores necesarios para su resolución, y a utilizar tales mediadores en la guía y control del comportamiento. El programa combina esta técnica con la resolución de problemas y el manejo de contingencias a lo largo de 16 sesiones, que incluyen actividades variadas, impersonales e interpersonales. En la resolución de situaciones interpersonales, se enfatiza la valoración de las distintas posibilidades de actuación ante una situación problemática, según los criterios de justicia, eficacia, seguridad y tipo de sentimientos que provoca. La instrucción de este programa se realiza mediante trabajo independiente o en grupo, desempeño de papeles, retroinformación, refuerzo y, especialmente, modelado del profesor o terapeuta y también de los compañeros $[28,29]$.

Otro foco de intervención ha consistido en desarrollar las habilidades socioemocionales de niños con TDAH o problemas de conducta. Un ejemplo sobresaliente es The Incredible Years Dinosaur Training, que, junto con el programa de intervención para padres (expuesto en el apartado anterior) y el programa para maestros, forma el paquete de intervención, multicontextual y multicomponente, para niños con problemas de conducta denominado The Incredible Years Program $[19,30]$.

El programa para niños de 4 a 8 años 'Escuela dinosaurio' comprende siete unidades en las que se trabajan los siguientes contenidos: importancia de las reglas; escuchar, pararse, pensar y comprobar; regulación emocional; pasos de la solución de problemas; técnica de la tortuga; amistad, compartir, trabajo en equipo; y escuchar, disculparse y hacer amigos. Los niños, en grupos de cinco o seis, durante unos seis meses acuden a 18-22 sesiones clínicas semanales fuera de la escuela. En la metodología utilizada, tiene un papel fundamental el uso del modelado en vídeo de niños enfrentándose a situaciones estresantes. Durante cada sesión, los niños ven en vídeo 10-12 secuencias cortas de niños padeciendo diferentes situaciones conflictivas o de estrés, discuten acerca de las secuencias y colaboran para resolverlas.

Como los niños pequeños son propensos a distraerse y tienen pocas habilidades organizativas, el programa incorpora estrategias específicas para motivarles y mantener su atención. Por ejemplo, se incluyen marionetas del tamaño de los niños en todas las sesiones, que participan con los grupos en la búsqueda de soluciones. También se utilizan cartas, cuadernos para colorear, dibujos, libros, fichas, juegos, pegatinas y premios para favorecer el aprendizaje. Un aspecto clave del éxito del programa es la estrecha colaboración con padres y profesores. De hecho, se mandan cartas semanales a ambos grupos explicándoles los conceptos clave y las habilidades importantes tratadas. Padres y profesores son informados también periódicamente sobre la buena conducta del niño.

Distintos trabajos (Tabla I) muestran la eficacia de este programa con niños con problemas de conducta o TDAH cuando se implementa sólo [31], y también junto con el programa para padres Incredible Years [32] y maestros [33]. En todos los casos se informa de importantes reducciones en los problemas externalizantes en casa y en la escuela, junto con mejoras en la identificación y regulación de emociones y en competencia social. También mejora la autoeficacia paterna y el manejo instruccional del maestro en el aula. En los análisis con muestras con TDAH, los padres informan igualmente de una disminución de la sintomatología básica del trastorno [31,32], manteniéndose los efectos un año después [34].

\section{Intervenciones con un enfoque socioconstructivista}

Desde un modelo de aprendizaje social, el desarrollo se produce por diferentes procesos mediados por la calidad de las interacciones del niño con los adul- 
Tabla II. Características y resultados de las diferentes intervenciones para preescolares con un enfoque socioconstructivista

\begin{tabular}{|c|c|c|c|c|c|c|}
\hline & Programas & Ámbito/edad & Agentes/duración & Contenidos & Metodología & Resultados \\
\hline $\begin{array}{l}\text { Barnett } \\
\text { et al [41] }\end{array}$ & $\mathrm{TM}$ & $\begin{array}{l}\text { Clases completas } \\
\text { 3-4 años }\end{array}$ & $\begin{array}{l}\text { Maestros } \\
1 \text { año }\end{array}$ & $\begin{array}{l}\text { Autorregulación, habilidades } \\
\text { matemáticas y lengua }\end{array}$ & $\begin{array}{l}\text { Mediación de pares } \\
\text { y profesores, juego }\end{array}$ & $\begin{array}{l}\downarrow \text { problemas de conducta } \\
\uparrow \text { habilidades académicas } \\
\text { (no significativamente) }\end{array}$ \\
\hline $\begin{array}{l}\text { Bodrova y } \\
\text { Leong [39] }\end{array}$ & $\mathrm{TM}$ & $\begin{array}{l}\text { Clases completas } \\
\text { 3-5 años }\end{array}$ & $\begin{array}{l}\text { Maestros } \\
3 \text { meses }\end{array}$ & $\begin{array}{l}\text { Andamiaje de escritura, } \\
\text { planes de juego }\end{array}$ & $\begin{array}{l}\text { Orientación a la acción, } \\
\text { mediadores externos, habla privada, } \\
\text { juego y planificación del juego, } \\
\text { actividades compartidas }\end{array}$ & 个 habilidades preacadémicas de lengua \\
\hline $\begin{array}{l}\text { Diamond } \\
\text { et al [43] }\end{array}$ & TM & $\begin{array}{l}\text { Clases completas } \\
\quad 5 \text { años }\end{array}$ & $\begin{array}{l}\text { Maestros } \\
1-2 \text { años }\end{array}$ & Autorregulación & $\begin{array}{l}\text { Habla privada, dramatizar } \\
\text { el juego, estrategias de } \\
\text { memoria y atención }\end{array}$ & $\begin{array}{l}\text { 个 funciones ejecutivas } \\
\text { y rendimiento académico } \\
\text { Mantenimiento y generalización de } \\
\text { los resultados en las actividades nuevas }\end{array}$ \\
\hline $\begin{array}{l}\text { Sonuga-Barke } \\
\text { et al [37] }\end{array}$ & NFPP & $\begin{array}{l}\text { TDAH } \\
3 \text { años }\end{array}$ & $\begin{array}{l}\text { Padres } \\
8 \text { semanas }\end{array}$ & $\begin{array}{l}\text { MC, mediación a través } \\
\text { del juego, interacciones } \\
\text { positivas, SP }\end{array}$ & $\begin{array}{l}\text { Discusión, juegos, desempeño de } \\
\text { papeles, modelado, deberes, diario } \\
\text { (enfermeras, no especialistas) }\end{array}$ & $\begin{array}{l}\text { No hay mejoras significativas en } \\
\text { la sintomatología básica de TDAH } \\
\text { (implementado por enfermeras, } \\
\text { no especialistas) }\end{array}$ \\
\hline $\begin{array}{l}\text { Thompson } \\
\text { et al [38] }\end{array}$ & NFPP & $\begin{array}{l}\text { TDAH } \\
4-5 \text { años }\end{array}$ & $\begin{array}{l}\text { Padres } \\
8 \text { semanas }\end{array}$ & $\begin{array}{l}\text { MC, relaciones positivas, } \\
\text { autorregulación }\end{array}$ & Mediación, juego & $\begin{array}{l}\downarrow \text { sintomatología de TDAH } \\
\text { Mantenimiento tras nueve semanas } \\
=\text { interacciones padres } / \text { niño }\end{array}$ \\
\hline
\end{tabular}

MC: modificación de conducta; NFPP: New Forest Parenting Package; SP: solución de problemas; TDAH: trastorno por déficit de atención/hiperactividad; TM: Tools Curriculum Programme.

tos dentro de la zona de desarrollo próximo. En este marco, el programa New Forest Parenting Package (NFPP) [10,35] subraya el importante papel que desempeñan las interacciones padres-hijos recíprocas y constructivas en la construcción de habilidades atencionales, del control de impulsos y de la autorregulación.

El NFPP se diseñó para preescolares con TDAH con la finalidad de reducir la sintomatología básica del trastorno y mejorar la competencia de los padres. Combina un entrenamiento tradicional a padres en técnicas de modificación de conducta con un componente novedoso dirigido a potenciar los procesos de interacción padres-hijos, que tienen un papel mediador en el desarrollo de habilidades de atención y autorregulación.

Sus objetivos son:

- Reducir las reacciones paternas negativas.

- Fomentar el establecimiento de límites.

- Incrementar cualitativa y cuantitativamente las interacciones padres-hijos.
- Potenciar la motivación y la estructura de las competencias atencionales y autorregulatorias mediante juegos y actividades.

Destaca por ser un enfoque naturalista aplicado en la propia casa con el objetivo de potenciar la generalización. La intervención se desarrolla en ocho sesiones de una hora, dirigidas por un profesional que proporciona retroalimentación y orientación a los padres.

El NFPP ha mostrado su eficacia en distintos ámbitos de la vida de los niños con TDAH (Tabla II). Sonuga-Barke et al [36] implementaron el programa con niños de 3 años con TDAH y obtuvieron disminuciones en los síntomas de TDAH y aumento del bienestar materno, que se mantuvieron 15 semanas después de tratamiento, y un 53\% del cambio clínicamente significativo. En cambio, cuando el mismo programa fue implementado por enfermeras no especializadas, no se encontraron mejoras significativas en la sintomatología de TDAH [37]. 
Una adaptación de este programa se ha aplicado también por Thompson et al [38], obteniendo en la reducción de los síntomas de TDAH tamaños del efecto comparables a los encontrados con medicación estimulante en niños más mayores.

Desde este mismo modelo socioconstructivista, el Tools of the Mind Curriculum Programme [39,40] plantea una visión ecológica del desarrollo de las funciones ejecutivas en el contexto del aula. El objetivo es enseñar a todos los niños preescolares, y especialmente a los que están en riesgo, a regular su comportamiento. A través del juego maduro e intencional se intentan potenciar tres funciones ejecutivas: memoria de trabajo, control inhibitorio y flexibilidad. Para ello se apoya en 60 actividades centradas en el currículo y en el uso de ayudas externas mediante interacciones interpersonales.

Un ejemplo de actividad para desarrollar la memoria de trabajo y la atención es el desempeño de papeles estructurado centrado en la lectura. Uno de los niños es el lector (y mantiene un dibujo de unos labios como recuerdo) y el otro es el receptor (tiene un dibujo de un oído). Mientras que el lector cuenta la historia, el receptor debe escuchar, con lo cual inhibe el deseo de hablar. Después de algunos meses de práctica, cada vez es menos necesaria la ayuda visual del dibujo. Se produce así el avance paulatino desde la actividad con los apoyos físicos de los dibujos, pasando por la escucha activa (donde el receptor hace preguntas al lector sobre el cuento) hasta llegar a la situación final, en la que la escucha activa se convierte en un aspecto internalizado del proceso lector. Otro ejemplo de actividad, en este caso para potenciar la función reguladora del lenguaje y, consecuentemente, la inhibición, es la colaboración de díadas en el recuento de objetos, donde solamente uno de los niños cuenta en voz alta y el otro actúa como 'controlador'. El controlador debe inhibir el deseo de contar y pensar metacognitivamente sobre si las respuestas son correctas. También se introduce una forma de juego dramático estructurado, donde los escenarios se planifican y se acuerdan de antemano. Como en casi todas las actividades, se subraya el papel del juego, acorde con la importancia que se le concede en la teoría vigoskiana. En este caso, el niño diseña de forma explícita un plan de juego que ha discutido con los compañeros previamente. Eso requiere inhibición de conductas inapropiadas para el papel, así como memoria de trabajo para representarlo, a pesar de la interferencia con otros juguetes $u$ otros papeles. Ayuda a potenciar la internalización de las reglas y a imponer límites al comportamiento para que se ajuste al papel desempeñado. Más específicamente, los niños de- ben mantener su propio papel y el de los demás compañeros en la mente (memoria de trabajo), inhibir el deseo de representar otros personajes (control inhibitorio) y ejercitar la flexibilidad para ajustarse a los turnos del diálogo (flexibilidad mental).

El grupo de Diamond [41-44] ha demostrado la efectividad del programa para promover las funciones ejecutivas y el rendimiento académico de preescolares en riesgo de 3 a 5 años.

\section{Conclusiones}

Las intervenciones con padres, solas o en conjunción con programas en la escuela o con el niño para potenciar habilidades académicas y autorregulatorias, han mostrado su eficacia para reducir la sintomatología de TDAH y los problemas académicos y socioemocionales en la etapa preescolar.

En contraposición, la investigación sobre los posibles factores que afectan a los resultados de las intervenciones es muy escasa. El éxito de los programas para padres está especialmente modulado por su disponibilidad para participar en las intervenciones, de manera que los resultados son menores o nulos cuando los padres no se implican en el tratamiento por conflictos laborales, estrés familiar o simplemente falta de motivación, relacionada, a veces, con la ausencia de un diagnóstico claro en el niño. Asimismo, la implementación y el mantenimiento de las estrategias enseñadas pueden variar en función de características de los padres; por ejemplo, los síntomas maternos de TDAH se relacionan con peores resultados [45]. Por el contrario, la gravedad de los síntomas de TDAH de los niños en la línea base se asocia con mejores resultados en las intervenciones. Otro aspecto que parece modular los resultados de los tratamientos es el grado de comunicación y colaboración entre terapeuta, padres y maestros. El fortalecimiento de la alianza terapéutica facilita el mantenimiento de las mejoras conductuales y la generalización de las habilidades adquiridas. Asimismo, los programas dirigidos a padres parecen tener una mayor efectividad cuando son implementados por especialistas que trabajan con preescolares [37-39].

La duración de los programas implementados con preescolares con TDAH también está, presumiblemente, influyendo en los resultados. En pocos casos las intervenciones que se han revisado superan las 10 sesiones [46], por lo que, al menos por el momento, no hay una respuesta clara sobre la extensión que deben tener para ser eficaces. Otra cuestión conectada con la anterior se refiere a la genera- 
lización de los resultados en el tiempo, habiéndose obtenido, en general, efectos positivos a corto plazo, pero que no se mantienen en evaluaciones de seguimiento realizadas a los dos años [26]. Dada la complejidad del TDAH y sus consecuencias a corto y largo plazo, el abordaje óptimo consistirá en implementar intervenciones tempranas, pero que se mantengan a lo largo del tiempo, y en las que participen plenamente las personas significativas de los distintos contextos en los que se desarrolla el niño.

Desde el contexto escolar, el modelo de respuesta a la intervención puede ser un procedimiento inclusivo válido de identificación e intervención temprana de alumnos con trastornos de atención y conducta. Este modelo, desarrollado en Estados Unidos a partir de la Ley de Educación para Personas con Dificultades, tiene un carácter, en esencia, preventivo, para lo que requiere, en primer lugar, identificar manifestaciones de TDAH. En segundo lugar, implica intervenir y controlar el progreso de los niños que presentan riesgo de padecer TDAH, y, por último, ir incrementando la intensidad de la intervención en función de la respuesta del alumno a ésta. Para implementar adecuadamente este modelo multinivel, los profesores de educación infantil tendrían que recibir una formación especial que les permitiera asumir más responsabilidades en el desarrollo de las habilidades de autorregulación de sus alumnos a través del currículo. Un enfoque tal se plasma en programas como 'Herramientas de la mente' [43] o pensamiento en voz alta [27]. Por último, tal y como subrayan algunos trabajos, habría que reforzar las competencias específicas preacadémicas en los paquetes de intervención en el contexto escolar [22].

\section{Bibliografía}

1. McDonnell MA, Glod C. Prevalence of psychopathology in preschool-age children. J Child Adolesc Psychiatr Nurs 2003; 16: 141-52.

2. Posner K, Melvin GA, Murray DW, Gugga SS, Fisher P, Skrobala A, et al. Clinical presentation of ADHD in preschool children: the Preschool ADHD Treatment Study (PATS). J Child Adolesc Psychopharmacol 2007; 17: 547-62.

3. Davis DW, Williams PG. Attention deficit/hyperactivity disorder in preschool-age children: issues and concerns. Clin Pediatr 2011; 50: 144-52.

4. Ghuman JK, Arnold LE, Anthony BJ. Psychopharmacological and other treatments in preschool children with attentiondeficit/hyperactivity disorder: current evidence and practice. J Child Adolesc Psychopharmacol 2008; 18: 413-47.

5. Greenhill LL, Posner K, Vaughan BS, Kratochvil CJ. Attention deficit hyperactivity disorder in preschool children. Child Adolesc Psychiatr Clin N Am 2008; 17: 347-66.

6. Wigal T, Greenhill L, Chuang S, McGough J, Vitiello B, Skrobala A, et al. Safety and tolerability of methylphenidate in preschool children with ADHD. J Am Acad Child Adolesc Psychiatry 2006; 45: 1294-303.

7. Daley D, Jones K, Hutchings J, Thompson M. Attention deficit hyperactivity disorder in pre-school children: current findings, recommended interventions and future directions. Child Care Health Dev 2009; 35: 754-66.

8. LaForett DR, Murray DW, Kollins SH. Psychosocial treatments for preschool-aged children with attention-deficit hyperactivity disorder. Dev Disabil Res Rev 2008; 14: 300-10.

9. McGoey KE, Eckert TL, DuPaul GJ. Early intervention for preschool-age children with ADHD: a literature review. J Emot Behav Disorder 2002; 10: 14-28.

10. Sonuga-Barke EJS, Thompson M, Abikoff H, Klein R, Brotman LM. Nonpharmacological interventions for preschoolers with ADHD. The case for specialized parent training. Infants Young Child 2006; 40: 402-8.

11. Young S, Amarasinghe JM. Practitioner review: non-pharmacological treatments for ADHD. A lifespan approach. J Child Psychol Psychiatry 2010; 51: 116-33.

12. Sanders MR, Markie-Dadds C, Turner KMT. Practitioner's manual for standard Triple P. Brisbane, Australia: Families International Publishing; 2001.

13. Jones K, Daley D, Hutchings J, Bywater T, Eames C. Efficacy of the Incredible Years basic parent training programme as an early intervention for children with conduct problems and ADHD. Child Care Health Dev 2007; 33: 749-56.

14. Triple P. URL: http://www.triplep.net. [19.11.2012].

15. Bor W, Sanders MR, Markie-Dadds C. The effects of the Triple-P Positive Parenting Program on preschool children with co-occurring disruptive behaviour and attentional/ hyperactive difficulties. J Abnorm Child Psychol 2002; 30: 571-87.

16. Hoath FE, Sanders MR. A feasibility study of enhanced group Triple P-Positive Parenting Program for parents of children with attention deficit/hyperactivity disorder. Behav Change 2002; 19: 191-206.

17. Markie-Dadds C, Sanders MR. Self-directed Triple P (Positive Parenting Program) for mothers with children at risk of developing conduct problems. Behav Cogn Psychother 2006; 34: 259-75.

18. Webster-Stratton C, Hancock L. Training for parents of young children with conduct problems: content, methods, and therapeutic processes. In Schaefer CE, Briesmeister JM, eds. Handbook of parent training. New York: John Wiley \& Sons; 1998. p. 98-152.

19. The Incredible Years. URL: http://www.incredibleyears.com. [19.11.2012].

20. Bywater T, Hutchings J, Daley D, Whitaker CI, Yeo ST, Jones K, et al. Long-term effectiveness of a parenting intervention for children at risk of developing conduct disorder. Br J Psychiatry 2009; 195: 318-24.

21. Williford AP, Shelton TL. Using mental health consultation to decrease disruptive behaviors in preschoolers: adapting an empirically-supported intervention. J Child Psychol Psychiatry 2008; 49: 191-200.

22. Kern L, DuPaul GJ, Volpe R, Sokol N, Lutz JG, Arbolino L, et al. Multi-setting assessment-based intervention for young children at-risk for ADHD: Initial effects on academic and behavioral functioning. School Psych Rev 2007; 36: 237-55.

23. Kern L, DuPaul GJ. Intervention for young children with, and at-risk for, ADHD. ADHD Report 2008; 16: 6-10.

24. DuPaul GJ, Kern L, Gormley MJ, Volpe RJ. Early intervention for young children with ADHD: academic outcomes for responders to behavioral treatment. School Ment Health 2011; 3: 117-26.

25. Barkley RA, Shelton TL, Crosswait C, Moorehouse M, Fletcher K, Barrett S, et al. Multi-method psycho-educational intervention for preschool children with disruptive behavior: preliminary results at post-treatment. J Child Psychol Psychiatry 2000; 41: 319-32.

26. Shelton TL, Barkley RA, Crosswait C, Moorehouse M, Fletcher K, Barrett S, et al. Multimethod psychoeducational intervention for preschool children with disruptive behavior: two-year post-treatment follow-up. J Abnorm Child Psychol 2000; 28: 253-66.

27. Camp BW, Bash M. Habilidades cognitivas y sociales en la infancia. Piensa en voz alta: un programa de resolución de problemas para niños. Valencia: Promolibro/CINTECO; 1998. 
28. Miranda A, Presentación MJ, Siegenthaler R, Jara P. Effects of a psychosocial intervention on the executive functioning in children with ADHD. J Learn Disabil 2011; Nov 7. [Epub ahead of print].

29. Presentación MJ, Siegenthaler R, Jara P, Miranda A Seguimiento de los efectos de una intervención psicosocial sobre la adaptación académica, emocional y social de niños con TDAH. Psicothema 2010; 22: 778-82.

30. Webster-Stratton C. The Incredible Years. The parents and children series. Seattle, EA: Seth Enterprises; 1999

31. Webster-Stratton C, Reid J, Hammond M. Social skills and problem- solving training for children with early-onset conduct problems: who benefits? J Child Psychol Psychiatry 2001; 42: 943-52.

32. Webster-Stratton C, Reid MJ, Beauchaine T. Combining parent and child training for young children with ADHD. J Clin Child Adolesc Psychol 2011; 40: 191-203.

33. Webster-Stratton C, Reid MJ, Hammond M. Treating children with early-onset conduct problems: intervention outcomes for parent, child, and teacher training. J Clin Child Adolesc Psychol 2004; 33: 105-24.

34. Webster-Stratton C, Reid MJ, Beauchaine T. One-year follow-up of combined parent and child intervention for young children with ADHD. J Clin Child Adolesc Psychol 2012; Sep 28. [Epub ahead of print].

35. Weeks A, Thompson M, Laver-Bradbury C. Information manual for professionals working with families with hyperactive children aged 2-9 years. Ashurst, UK: Ashurst Child \& Family Evidence Centre; 1999.

36. Sonuga-Barke EJS, Daley D, Thompson M, Laver-Bradbury C, Weeks A. Parent-based therapies for preschool attentiondeficit/hyperactivity disorder: a randomized, controlled trial with a community sample. J Am Acad Child Adolesc Psychiatry 2001; 40: 402-8.

37. Sonuga-Barke EJS, Thompson M, Daley D, Laver-Bradbury C.
Parent training for pre-school attention-deficit/hyperactivity disorder: Is it effective when delivered as routine rather than as specialist care? Br J Clin Psychol 2004; 43: 449-57.

38. Thompson MJ, Laver-Bradbury C, Ayres M, Le Poidevin E, Mead S, Dodds C, et al. A small-scale randomized controlled trial of the revised New Forest Parenting Programme for preschoolers with attention deficit hyperactivity disorder. Eur Child Adolesc Psychiatry 2009; 18: 605-16.

39. Bodrova E, Leong DJ. Tools of the mind: the Vygotskian approach to early childhood education. 2 ed. Columbus, OH: Merrill/Prentice Hall; 2007.

40. Tools of the Mind. URL: http://www.toolsofthemind.org. [19.11.2012].

41. Barnett WS, Jung K, Yarosz DJ, Thomas J, Hornbeck A, Stechuk R, et al. Educational effects of the Tools of the Mind curriculum: a randomized trial. Early Child Res Q 2008; 23: 299-313.

42. Bodrova E, Leong DJ. Tools of the mind: a case study of implementing the Vygotskian approach in American early childhood and primary classrooms. UNESCO Innodata Monographs: Educational Innovations in Action No. 7. Geneva: International Bureau of Education, UNESCO; 2001.

43. Diamond A, Barnett WS, Thomas J, Munro S. Preschool program improves cognitive control. Science 2007; 318: 1387-8.

44. Diamond A, Lee K. Interventions shown to aid executive function development in children 4 to 12 years old. Science 2011; 333: 959-64.

45. Sonuga-Barke EJS, Daley D, Thompson M. Does maternal $\mathrm{ADHD}$ reduce the effectiveness of parent training for preschool children's ADHD? J Am Acad Child Adolesc Psychiatry 2002; 41: 696-702.

46. Beauchaine TP, Webster-Stratton C, Reid M. Mediators, moderators, and predictors of 1-year outcomes among children treated for early-onset conduct problems: a latent growth curve analysis. J Consult Clin Psychol 2005; 73: 371-88.

\section{Moderators of psychosocial intervention in preschoolers with attention deficit hyperactivity disorder}

Introduction. Although the diagnosis of attention deficit hyperactivity disorder in preschoolers is increasingly common, relatively little is known about the treatment in this developmental period. Some side effects of pharmacological intervention discourage its use as first-line intervention at this age.

Aim. To analyze the effectiveness of psychosocial interventions designed to respond to the needs presented by these children, especially those based on cognitive-behavioral and socio-constructivist models.

Development. The review highlights the effectiveness of parent training programs, alone or combined with interventions at school and with the children. Communication between parents and teachers has been shown to be a determinant of their success. Another aspect that seems to favor the efficacy of treatment in this stage of education is the inclusion in the school curriculum of mediated activities aimed at developing self-regulation.

Conclusions. In preschoolers with attention deficit hyperactivity disorder, preventive action based on these models, increasing the intensity of the intervention depending on the student's response, can help to avoid future problems.

Key words. ADHD. Cognitive-behavioral. Modulating factors. Preschoolers. Psychosocial intervention. Socio-constructivist. 